\title{
Supportive Care in Hematology
}

\author{
John Hiemenz, MD \\ and Reinhold Munker, MD
}

\section{CONTENTS}

INTRODUCTION

Diagnosing Suspected Infection in Neutropenic

Patients

Treating Infections in Neutropenic Patients

Prophylaxis of Infections in Neutropenic Patients

Venous Access

FurTher CONSIDERATIONS

REFERENCES

\section{INTRODUCTION}

Patients with hematological malignancies such as leukemias and lymphomas are predisposed to a wide spectrum of infections that need special attention. These patients are immunosuppressed not only as a result of the immune defects associated with the underlying disease, but also because of the treatment regimens that generally further decrease the patient's resistance to infections. An intensive transfusion support with platelets, red cell concentrates, immunoglobulins, cytokines, and other drugs is necessary. Many patients also need antiemetic agents, nutritional support, pain medication, and, very often, venous catheters. All of these measures are considered supportive care and apply in a similar fashion to the high-dose treatment of solid tumors. Supportive care measures, along with the treatment and prophylaxis of infections, are discussed in this chapter. Transfusion support is discussed in Chapter 22. 


\section{DIAGNOSING SUSPECTED INFECTION IN NEUTROPENIC PATIENTS}

The immune system can be compromised by the underlying disease, the treatment of the disease, or many procedures and devices utilized in support of treatment of the underlying disease (e.g., central venous catheters). Neutropenia has been recognized as the major risk factor for development of opportunistic infection in patients with hematological malignancy or undergoing cancer chemotherapy. Bodey and colleagues studied the relationship between the neutrophil count and the development of severe infection in 52 patients treated for acute leukemia at the National Institutes of Health from 1959 to 1963 (1). In the presence of severe neutropenia, defined as less than 100 cells $/ \mathrm{mm}^{3}$, there were 43 episodes per $1000 \mathrm{~d}$ with a marked drop in infection rate as the neutrophil count rose. In addition, the risk of infection increased with increasing duration of neutropenia. This relationship between neutropenia and life-threatening infection has been reported by numerous investigators since the initial publication by Bodey et al. in $1966(2,3)$. The Infectious Diseases Society of America (IDSA) defines neutropenia as an absolute neutrophil count of less than 500 cells $/ \mathrm{mm}^{3}$, or $<1000$ cells $/ \mathrm{mm}^{3}$ with a predicted decrease to less than $500 \mathrm{cells} / \mathrm{mm}^{3}$ (4). Although the risk of neutropenia has been recognized for more than $40 \mathrm{yr}$, other qualitative defects in humoral and/or cellular immunity may also increase the risk of infection with a variety of pathogens including bacterial, fungal, and viral pathogens. Examples include infections with encapsulated bacteria in patients with dysgammaglobulinemia due to underlying myeloma or infections due to cellular immune deficiency such as Pneumocystis carinii pneumonia or cryptococcal meningitis in the patient with Hodgkin's lymphoma, and invasive aspergillosis or cytomegalovirus (CMV) pneumonia in the leukemic patient with chronic graft-vs-host disease (GVHD) on immunosuppressive therapy (5). All of these patients may have a normal or elevated neutrophil count.

When faced with fever in the neutropenic patient, defined by the IDSA as a single oral temperature of $38.3^{\circ} \mathrm{C}\left(101.0^{\circ} \mathrm{F}\right)$ or $38.0^{\circ} \mathrm{C}\left(100.4^{\circ} \mathrm{F}\right)$ for $1 \mathrm{~h}$ or longer, the diagnosis of severe infection must be considered. This situation is consider an emergency and should lead to prompt evaluation and initiation of empirical antimicrobial treatment. Evaluation includes a thorough history and physical examination (4). The history should include the nature and status of the underlying disease, chemotherapy received, other immunosuppressive drugs such as steroids, use of cytokines, and past and recent infections as well as previous procedures. Initial examination should assess all sites of possible infection. The profoundly neutropenic patient may have minimal signs or symptoms of inflammation with 50 to $75 \%$ of cases having no definable source of infection when fever develops. The clinician should carefully inspect the eyes, orophar- 
ynx, and skin (with particular attention to vascular catheter tunnel and exit sites, bone marrow aspirate sites, nails, and nailbeds) (2). The perineal and perirectal areas are frequently missed but are critical areas to inspect initially and on a regular basis when fever persists. Evidence of perirectal or perianal infection in the neutropenic patient may require modification of initial empirical antimicrobial treatment. Surgical intervention may be warranted for drainage of developing abscess(es) (6). It is also important to carefully inspect the scalp, as hair may hide evidence of infection.

In addition to a thorough physical examination, laboratory evaluation of the febrile neutropenic patient includes a hemogram, electrolytes, renal, and liver function tests. Cultures of blood and urine should be obtained, as these are the most common sites of microbiologically documented infection. A minimum total of two sets (aerobic and anaerobic) of blood cultures should be obtained for the initial evaluation. It has been recommended that, in addition to drawing one set of cultures from a peripheral venipuncture, one set of blood cultures should be obtained from each lumen in patients with central venous catheters. In this group of patients, it is often difficult to obtain peripheral venous access even for simple blood sampling. The need to routinely obtain cultures from the peripheral vein in cancer patients with central venous catheters, therefore, has often been questioned. In a recent retrospective review, DeJardin et al. found a high predictive value of negative blood cultures drawn from central venous catheters of febrile cancer patients (7). These authors have suggested that peripheral venipuncture was thus not routinely required unless blood cultures from the central venous catheter were found to be positive. A routine chest radiograph should be performed to exclude or diagnose pneumonia. Performance of the chest radiograph, however, should not delay initiation of empirical antibacterial therapy immediately after cultures are obtained.

Further diagnostic steps should be taken if clinically indicated. In addition to blood and urine, cultures should be obtained from any other site(s) found on exam to be suspicious for possible focus of infection. If skin lesions are present, biopsy or aspiration should be performed. Biopsy specimens should then be sent for cytology and/or histopathology with special stains for microorganisms as well as culture for bacteria, fungi, and viruses (e.g., Herpes simplex and Varicella zoster). In the setting of diarrhea, stool culture for bacterial pathogens, screening for the presence of Clostridium difficile toxin, as well as evaluation for ova and parasites should be considered. Cerebrospinal fluid (CSF) should be obtained from a lumbar puncture (or Ommaya reservoir when present) in patients who have neurological symptoms or a change in mental status to diagnose meningitis. Bacterial surveillance cultures from the oropharynx, urine, and stool have been performed on a regular basis in neutropenic patients at a number of institutions. Although information obtained from these cultures may be valuable for epide- 
miological purposes, they are not recommended for the management of the individual patient. In the absence of specific signs or symptoms of infection, bacterial surveillance cultures have little value in predicting infection, rarely change antibiotic therapy, and are costly (2).

High-resolution computed tomography (CT) of the chest should be considered in any patient with abnormalities on routine chest radiograph to better define possible pulmonary infection. CT scan should also be considered despite negative routine radiograph of the chest if symptoms or signs of pulmonary infection exist such as cough, shortness of breath, or hypoxemia, or if there is persistent or recurrent fever after a week of broad spectrum empirical antibacterial therapy (8). Bronchoscopy with bronchoalveolar lavage (BAL) should be performed in patients with abnormal radiograph or CT scan to obtain specimens for cytology, culture, and stains for bacteria, fungal, and viral pathogens. If viral pneumonia is suspected, specimens may be examined for viral DNA by PCR.

Tests for inflammatory cytokines such as C-reactive protein, procalcitonin, interleukin-6, and interleukin-8 may correlate with the presence of an infection in neutropenic patients, but none are specific or reliable and they cannot be used for treatment decisions in the acute situation. The presence of galactomannan in blood has been utilized for a number of years in Europe as a surrogate marker of invasive aspergillosis and was recently approved for use in the United States by the Food and Drug Administration for a similar indication. The sensitivity and specificity of this test may vary in different populations of patients (i.e., children versus adults, neutropenic versus non-neutropenic hosts) and therefore results must be taken in context with the clinical situation. $\beta$-glucan is another surrogate marker for invasive fungal infection and may apply to a broad group of pathogens that possess a high concentration of $\beta$-glucan in their cell wall (9-11).

\section{TREATING INFECTIONS IN NEUTROPENIC PATIENTS}

A large array of pathogens is responsible for infections in immunosuppressed and/or neutropenic patients with hematological disorder. The source of infection may be from the normal endogenous flora or may result from nosocomial acquisition from the exogenous environment. Gram-negative rods (Escherichia coli, Klebsiella pneumoniae, Pseudomonas aeruginosa, Enterobacter spp., and others) usually arise from the gastrointestinal tract and may cause serious infections in compromised patients. Antimicrobial developments in the 1980s and 1990s led to an increase in the armamentarium of antimicrobial agents available to treat these common Gram-negative pathogens (e.g., extended-spectrum $\beta$-lactams, carbapenems, quinolones) (2,4). Unfortunately, increased exposure to these agents empirically and prophylactically has led to the selection of Gram-negative pathogens with broad-spectrum antimicrobial resistance. Organisms with broad 
antibacterial resistance such as Acinetobacter anitratis and Stenotrophomonas maltophilia are usually acquired from the hospital environment $(3,12,13)$. The incidence of infection with Gram-positive cocci (Coagulase-negative staphylococci, Staphyloccocus aeurus, $\alpha$-hemolytic such as Streptococcus mitis, and Enterococcus spp.) has increased over the last decade, and now accounts for the majority of microbiologically documented bacterial infections in the neutropenic host in many institutions $(3,12,13)$. Although the increased use of indwelling central venous silastic catheters has been considered to be the cause of the increase in Gram-positive bacteria infections, the oropharnynx and gastrointestinal tract are also considered sources of infection. Severe mucositis after intensive chemotherapy and the use of quinolone prophylaxis have been found to be independent risk factors that can increase the risk of $\alpha$-hemolytic streptococcal sepsis. Bacteremia with $\alpha$-streptococcus can lead to septic shock accompanied by adult respiratory distress syndrome (ARDS), more commonly seen with Gramnegative rod bacteremia (14). Approximately $10-20 \%$ of neutropenic patients with microbiologically documented bacterial infections are infected with anerobic bacteria (anaerobic streptococci, Clostridia spp., Bacteroides spp.) or mixed (Gram-positive, Gram-negative, aerobic, and/or anaerobic) infections.

All neutropenic and febrile patients are at risk of fungal infections. Patients with T-cell dysfunction caused by an underlying hematological disorder or its treatment may also be at increased risk for fungal infection. GVHD after allogeneic blood or marrow transplantation is a well-known risk factor for invasive mycosis that may last for months or years (15-17). The use of monoclonal antibodies with anti-T-cell activity for treatment of lymphoproliferative malignancies or GVHD has been recognized as a risk factor for development of invasive fungal infections (18). Candida albicans and Aspergillus spp. have accounted for the majority of invasive fungal infections in patients with hematological disorders. However, a shift toward the non-albicans Candida spp. and an increase of Aspergillus spp. as the major cause of fungal-related morbidity and mortality has been seen in many institutions (19). In addition, infection with a wide variety of previously uncommon opportunistic fungal pathogens has been noted over the last two decades. These emerging pathogens include a number of septate filamentous fungi such as Fusarium spp. and Scedosporium spp. that may mimic Aspergillus spp. on microscopic inspection of tissues. Nonseptate filamentous fungal infections with Zygomycetes, previously considered a complication of uncontrolled diabetes, has been increasing reported in patients with hematological malignancies or after hematopoietic stem cell transplantation. Invasive infections with the dematiaceous molds, as well as a variety of yeast including the Trichosporon spp. have also been reported. It is important to be familiar with these potential pathogens, as they may have variable sensitivities 
or be frankly resistant to amphotericin $\mathrm{B}$, the newer triazoles, and/or the echinocandins (20).

Viral infections in patients with hematological disorders are most commonly the consequence of reactivation of members of the herpesviruses (H. simplex, $V$. zoster, CMV, HHV-6, etc.) and will be discussed in a later chapter. Community respiratory viruses such as influenza, parainfluenza, adenovirus, respiratory syncytial virus (RSV), and rhinovirus have been reported to cause outbreaks of infection in highly susceptible patients and must be taken into account when considering antiviral therapy and infection control practices for a hematology and/or transplant clinic or ward (21-23). Adenovirus and polyoma type BK virus have been associated with hemorrhagic cystitis, particularly after hematopoietic stem cell transplantation. Less common viral pathogens include echovirus, cocksackie virus, rotavirus, polyoma type JC virus, and parvovirus B19 and should be considered in the appropriate setting.

\subsection{Empirical Treatment}

In patients with neutropenia $\left(<500\right.$ neutrophils $\left./ \mathrm{mm}^{3}\right)$ and unexplained fever $\left(\geq 38.3^{\circ} \mathrm{C}\right.$ ), broad-spectrum antibiotic treatment should be started without delay. Although recent studies suggest that patients with fever and neutropenia classified as "low risk" may be treated with ciprofloxacin and amoxicillin-clavulanate through the oral route $(24,25)$, most would consider patients with hematological disorder at "high risk" requiring hospitalization and intravenous antimicrobial therapy. Historically, the classic therapy to begin with was a combination of an aminoglycoside with an extended-spectrum penicillin. Extensive clinical trials and more recent IDSA guidelines, however, suggest the following options:

- Monotherapy with either an extended-spectrum cephalosporin with activity against $P$. aeruginosa such as cefepime or ceftazidime.

- Another option for monotherapy is to use a broad-spectrum carbapenem, with activity against $P$. aeruginosa, such as imipenem/cilistatin or meropenem.

- Combination therapy may still be used as initial therapy for the febrile neutropenic patient. The IDSA recommends the routine addition of an aminoglycoside when treating with an antipseudomonal penicillin, although there have been recent data that support the use of piperacillin/tazobactam as a single agent $(4,27)$. When combination therapy is felt to be indicated, an aminoglycoside plus either an antipseudomonal penicillin, cephalosporin (cefepime or ceftazidime), or carbapenem (imipenem/cilistatin or meropenem) is recommended. In patients with suspected- or proven-resistant Gram-positive bacterial infections, vancomycin should be combined with any one of cefepime, ceftazidime, imipenem/cilistatin, meropenem (with or without an aminoglycoside), or an aminoglycoside plus an antipseudomonal penicillin (Table 1). 
Table 1

Empirical Treatment of Fever of Unknown Origin in a Neutropenic Patient

A. Single-agent treatment

Extended-spectrum cephalosporin (cefepime or ceftazidime)

Carbapenem (imipenem/cilistatin or meropenem)

B. Combination treatment

Extended-spectrum penicillin + aminoglycoside

Extended-spectrum cephalosporin (cefepime or ceftazidime) + aminoglycoside

Carbapenem (imipenem/cilistatin or meropenem) + aminoglycoside

Extended-spectrum penicillin + aminoglycoides + vancomycin

Extended-spectrum cephalosporin (cefepime or ceftazidime) or carbapenem

(imipenem/cilistatin or meropenem) + vancomycin

Examples of patients at risk of having resistant Gram-positive infection include those suspected of catheter infection based on clinical findings, those colonized with methicillin-resistant $S$. aureus (MRSA) or penicillin- or cephalosporinresistant pneumococci, and those with documented Gram-positive bacteremia before antimicrobial susceptibility has been defined. Vancomycin should also be considered as part of early empirical antibacterial therapy in patients with hypotension or cardiovascular instability. Fever and neutropenia should be considered a dynamic process with continued and ongoing evaluation of the patient. Antimicrobial therapy should be adjusted based on culture results, the clinical course of the patient, and persistence of fever.

With persistent or recurrent fever in the neutropenic patient after 3 to $5 \mathrm{~d}$ of broad-spectrum antibacterial coverage without a documented source of infection, the empirical addition of broad-spectrum antifungal coverage is considered the standard of care to reduce the risk of morbidity and mortality caused by invasive mycosis (28). Although overall survival was not improved in the studies performed at the National Cancer Institute and by the European Organisation for Research and Treatment of Cancer (EORTC) more than two decades ago, the incidence of documented invasive fungal infections was significantly reduced when amphotericin B deoxycholate was empirically added to ongoing antibacterial therapy $(28,29)$. Over the last $10 \mathrm{yr}$, a number of alternative antifungal agents have been shown to be equally effective yet less toxic as compared with amphotericin B for empirical therapy of the persistently febrile neutropenic patient. These include members of the class of lipid formulations of amphotericin B, extended spectrum triazoles, and the echinocandins (30-34) (Table 2). Attempts to make a definitive diagnosis of infection should continue with thorough examination, cultures, and chest radiography including $\mathrm{CT}$ of the lungs with 
Table 2

Empirical Antifungal Therapy for Patients With Persistent Fever and Neutropenia

A. Polyenes

Amphotericin B deoxycholate

Lipid formulations of amphotericin B

Liposomal amphotericin (AmBisome) ${ }^{a}$, amphotericin B complex (Abelcet)

B. Azoles

Itraconazole (intravenous formulation with cyclodextran) ${ }^{a}$

Voriconazole (intravenous and oral formulation)

C. Echinocandins

Caspofungin $^{a}$

\footnotetext{
${ }^{a}$ Approved for this indication by the Food and Drug Administration.
}

bronchoscopy and BAL if abnormal (8). Serological tests for evidence of invasive fungal infection using galactomannan and $\beta$-glucan may be helpful; however, false-positive and false-negative tests have been noted (9-11).

\subsection{Documented Bacterial Infections}

Bacterial infections should be treated according to the antibiotic spectrum and the clinical pattern of sensitivity. A marked increase in antibiotic resistance has occurred over the last decade. The most common resistant bacteria encountered in patients with hematological disorders include MRSA, methicillin-resistant $S$. epidermidis, vancomycin-resistant enterococcus (VRE), and $\beta$-lactamase producing Gram-negative bacilli $(12,13)$. The National Nosocomial Infections Surveillance System (NNIS) noted a steady increase in MRSA to more than 50\% of isolates from 300 hospitals reported to the database in 1999 and over 55\% MRSA in $2000(35,36)$. Although viridans streptococci are found as part of the normal microbial flora, they have been isolated as pathogens with increasing frequency in patients with hematological disorders. Risk factors include severe neutropenia, mucositis, treatment with high-dose cytosine arabinoside, and antimicrobial prophylaxis with either a fluoroquinolone or trimethoprimsulfamethoxazole. Although most patients respond to therapy, a toxic shock-like syndrome with hypotension, a maculopapular rash, palmar desquamation, and ARDS has been reported in as many as $25 \%$ of cases $(15,37)$.

The increased use of vancomycin for the treatment of proven or probable MRSA, viridans streptococci, or for empirical therapy of febrile neutropenic patients is considered to be one of the major risk factors for the increasing incidence of VRE seen in the 1990s. Other risk factors noted have been the use of oral vancomycin for the treatment of $C$. difficile-related enteritis, use of drugs with anaerobic activity, gastrointestinal procedures, mucositis, acute renal fail- 
ure, glucose intolerance, or diabetes (38-40). Compared with the mean rate of growth of vancomycin-resistant isolates of enterococci reported to the NNIS database over the prior $5 \mathrm{yr}$, the rate of growth between 1998 and 2000 appeared to be decreasing $(35,36,41)$. It is hoped that this trend will continue as a result of both better infection control practices and more judicious use of vancomycin. Newer antimicrobials such as linezolid and quinupristin/dalfopristin have been developed for the treatment of $\operatorname{VRE}(42,43)$. Although the initial experience had suggested that linezolid might have more hematological side effects (especially thrombocytopenia) compared with the other antimicrobial agents, recent reports in both children and adults suggest that the toxicity profile is not worse than that of vancomycin $(44,45)$. Even with these newer agents, however, resistant organisms have already been reported (46).

Most Gram-negative bacterial isolates from patients with hematological disorders remain sensitive to standard antibacterial agents. Patients will usually respond to appropriate therapy if there is eventual recovery from immune dysfunction. As with the Gram-positive bacteria, however, there has been a gradual increase in isolation of multi-drug resistant Gram-negative organisms like $S$. maltophilia (12). These infections usually occur in the most complicated patients with uncontrolled malignancy, prolonged hospitalization, prolonged immune suppression, and multiple courses of prior antibiotic therapy.

\subsection{Documented Fungal Infections}

Oropharyngeal candidiasis is often diagnosed clinically with classical signs of erythema, white plaques, and ulcers. Presumptive diagnosis of infection with Candida spp. by inspection only, without examination of wet mount or Gram stain of exudate material, may not be accurate. Infection with $H$. simplex, bacterial infections, and noninfectious causes of mucositis in patients with underlying hematological disorders may mimic oral lesions due to Candida spp. Local treatment can be attempted with nonresorbable antifungal agents (nystatin or clotrimazole), but if this fails, or if the patient is severely neutropenic, systemic treatment may be indicated. For patients not already receiving azole prophylaxis, fluconazole is the most commonly used antifungal agent for the systemic treatment of oropharyngeal candidiais. Typically, this infection is due to $C$. albicans, which is usually highly susceptible to treatment with fluconazole. In the unusual setting of oropharyngeal infection refractory to systemic therapy with fluconazole, documentation of persistent or recurrent fungal infection should be attempted. Treatment with a broader-spectrum antifungal agent that covers the non-albicans species of $\mathrm{Can}$ dida would then be recommended. Broader-spectrum antifungal drugs would include amphotericin B, one of its lipid formulations, or an echinocandin. Although alternative triazole such as itraconazole or voriconazole could be considered, cross resistance between azoles has been seen among the non-albicans Candida spp. 
Most experts would therefore recommend using either an echinocandin or amphotericin B product in this setting. Esophageal candidiasis is common in patients with AIDS and hematological neoplasms undergoing chemotherapy. A typical symptom is a burning pain on swallowing. Esophageal candidiasis can also be treated with local or systemic antifungal therapy. If the patient has already been on fluconazole prophylaxis, or has failed systemic therapy with fluconazole, the same attempts to document persistent fungal infection and switch to alternative therapy with an echinocandin or amphotericin product are recommended.

Deep-seated or invasive candidiasis is a spectrum of infections. It may present as isolated candidemia, infection of a single organ (endocarditis or endophtalmitis), or wide-spread disseminated infection involving the liver and spleen (hepatosplenic candidiasis), kidneys, and/or other organs. Some clinicians will differentiate disseminated candidiasis into acute and chronic forms. Acute disseminated candidiasis is a syndrome of acute, life-threatening infection presenting as persistent fungemia, hypotension, and multi-organ failure in the neutropenic patient. $\mathrm{Cu}-$ taneous and skeletal muscle involvement frequently occurs in the acute form of invasive candidiasis. In contrast, chronic disseminated candidiasis is established by hematogenous spread of infection during neutropenia. It is not accompanied by hypotension and frequently previous blood cultures failed to document fungemia prior to the patient manifesting signs and symptoms of infection. As marrow function recovers and neutropenia resolves, radiological manifestations of chronic infection are seen in the liver and spleen on ultrasound, CT, or most reliably on magnetic resonance imaging (MRI). The patient may have presented with persistent or new fevers, but may also have had minimal temperature elevations with anorexia, weight loss, or failure to thrive along with an isolated increase in alkaline phosphatase. This clinical presentation warrants further evaluation to rule out chronic disseminated fungal infection. Although frequently referred to as "hepatosplenic candidiasis" because of the radiological manifestations in the liver and spleen, this infection may be widely disseminated involving many other organs. Confirmation of the diagnosis of fungal infection with biopsy and culture of liver and/or other accessible lesions should be attempted in order to direct appropriate therapy. Although most of these infections are caused by Candida spp. disseminated infection with other bacterial, fungal, or protozoal pathogens may have similar clinical presentations $(47,48)$.

Disseminated candidiasis requires prompt and aggressive therapy. Sources for Candida fungemia include not only indwelling central venous catheters, but also the gastrointestinal tract. The need for routine removal of central venous silastic catheters (Hickman ${ }^{\circledR}$ and Broviac ${ }^{\circledR}$ [C.R. Bard, Inc.], etc.) for successful treatment of candidemia has therefore been controversial. Fungemia with certain species of Candida (e.g., C. parapsilosis) however is clearly felt to be linerelated, necessitating removal of the indwelling catheter. If the catheter is left in 
place and fungemia does not rapidly clear with appropriate antifungal therapy, the central line will need to be removed promptly. All patients with positive blood cultures for Candida spp. should be treated with an appropriate course of antifungal therapy regardless of immune status and whether or not the central line was removed $(49,50)$.

Isolated candidemia can be successfully treated with 10-14 d of antifungal therapy after clearing of fungemia. Historically, however, appropriate treatment for disseminated candidiasis consisted of prolonged treatment with amphotericin B, possibly combined with flucytosine. Fluconazole is much better tolerated for longer courses of therapy. The use of this azole has usually been reserved for the non-neutropenic patient and as follow-up therapy for patients responding to induction treatment with amphotericin B. Lipid formulations of amphotericin have been shown to be useful in patients with disseminated disease refractory to treatment with conventional amphotericin B and fluconazole (47). A recent randomized trial comparing caspofungin with amphotericin B deoxycholate in the treatment of invasive candidiasis has shown the echinocandin to be equally efficacious and less toxic than the polyene in both neutropenic and nonneutropenic hosts (51).

Infection with Aspergillus spp., once the second most-common fungal infection in immunocompromised hosts, is now the most common cause of mortality related to invasive fungal infections in the United States (19). More than two decades ago, Gerson et al. showed that a prolonged severe neutropenia ( $>2 \mathrm{wk}$ ) led to an exponential rise in risk of invasive pulmonary aspergillosis in a group of patients with leukemia (52). More recent studies have focused on the risk of invasive fungal infections in the allogeneic transplant recipient on immunosuppressive therapy for GVHD after marrow recovery $(15,53)$. The most common site of invasive Aspergillus infection is the lungs. Infections can also involve the sinuses and other organs and expand in a locally destructive fashion. Pulmonary disease has a characteristic radiological appearance (round mass overlaid by a crescent of air) (8) and may result in catastrophic bleeding. On CT, a nodular lesion is typical and has a surrounding "halo" due to bleeding into the tissues. These lesions will frequently evolve as neutropenia resolves with contraction of tissue surrounded by a crescent of air ("air crescent" sign) (see Fig. 3.1A,B). Although amphotericin B deoxycholate had been the drug of choice for invasive Aspergillus infections for four decades, overall response to treatment was poor in patients with underlying hematological disorders with most neutropenic patients dying of fungal disease $(48,53,54)$. Over the last decade, lipid formulations of amphotericin B have become available, allowing higher doses of drug to be delivered safely for longer periods of time (55). Caspofungin was also found to be active in a retrospective trial of the treatment of invasive aspergillosis in patients refractory or intolerant to other standard antifungal therapy (mainly amphotericin B or its lipid formulations). A recent large, multinational, multi- 

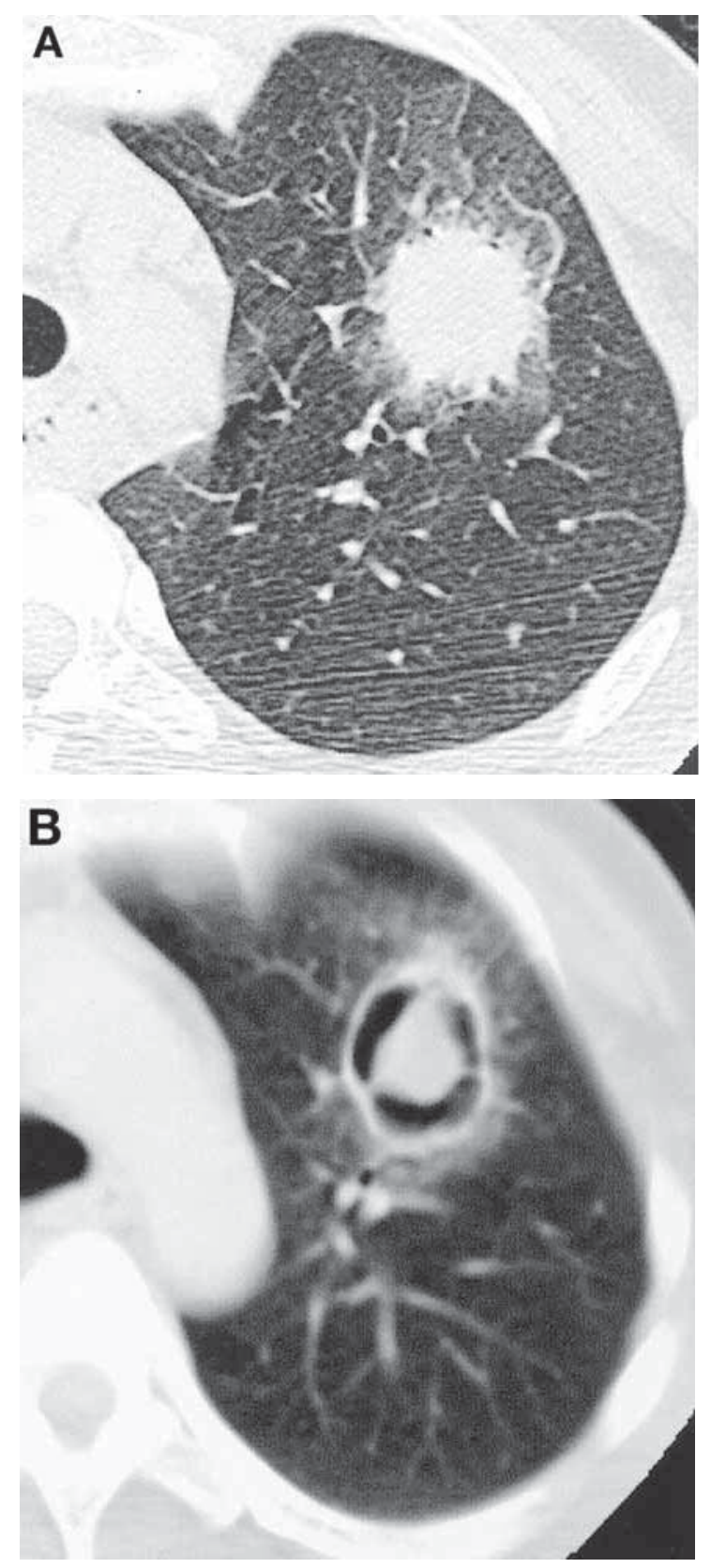

Fig. 3.1. Invasive pulmonary aspergillosis (IPA) in a neutropenic patient with acute myelogenous leukemia. Above, a typical "air halo" can be seen on CT imaging with surrounding ground glass opacity. The patient was starting on antifungal therapy, recovered his blood counts, and developed an "air crescent" sign (see lower image) $10 \mathrm{~d}$ later. The diagnosis of IPA was confirmed after surgical resection. (Reproduced from ref. 8 , with permission). 
institutional randomized clinical trial has led to a major change in the recommended standard therapy for invasive aspergillosis. In this trial, amphotericin B deoxycholate was compared prospectively to the newer triazole voriconazole in patients with proven or probable invasive aspergillosis. This study showed superior response rates and overall outcome in patients who began therapy with voriconazole as compared with those whose treatment was initiated with amphotericin B deoxycholate. This superior outcome was seen even when taking in to account a change to other licensed antifungal therapy, which in the case of amphotericin B deoxycholate, was most commonly a switch to a lipid formulation of amphotericin B because of toxicity. Moreover, the survival benefit with voriconazole was seen even in the neutropenic patient population and those who had received a hematopoietic stem cell transplantation (56). Although one must take into account the increased risk of drug-drug interactions, visual hallucinations, and photosensitivity of the skin not seen with the more narrow-spectrum agent fluconazole, voriconazole now appears to be the drug of choice for the treatment of invasive aspergillosis in the immunocompromised host with a hematological disorder. Unfortunately, a large proportion of these patients were still considered nonresponders and no doubt died from complications of invasive aspergillosis. Although difficult to study in a randomized trial, most experts feel that surgical debridement, when possible, is beneficial in improving overall outcome in patients with invasive filamentous fungal infections like Aspergillus $(54,57)$.

The availability of newer agents with activity against Aspergillus spp. has led to the consideration of combination therapy for treatment of invasive disease in the immunocompromised host. A number of in vitro studies, in vivo animal models, and retrospective clinical reports have suggested the potential benefit of combination therapy, particularly utilizing a cell wall-active agent such as an echinocandin with a cell membrane-active drug, either voriconazole or a polyene (58-64). Marr et al. suggested an improved outcome with the routine addition of caspofungin to voriconazole in a group of bone marrow transplant recipients treated for invasive aspergillosis at the Fred Hutchinson Cancer Research Center in Seattle. Unfortunately, this was not a randomized trial and long-term follow up of these patients is unknown (65). The continued and increasing importance of infections with Aspergillus spp. warrant further prospectively controlled trials of combination therapy.

C. albicans and Aspergillus spp. have accounted for the majority of opportunistic invasive fungal infections in patients with hematological disorders. In addition to a shift toward the non-albicans Candida spp. and an increase of Aspergillus spp. as a major cause of morbidity and mortality in many centers, a wide variety of previously uncommon opportunistic fungal pathogens have been encountered over the last two decades (19). These emerging pathogens include 
septate filamentous fungi such as Fusarium spp. that may be difficult to distinguish from Aspergillus spp. on microscopic inspection of tissues, along with an expanding group nonseptate Zygomycetes, the dematiaceous molds, as well as a variety of yeasts including the Trichosporon spp.. The increasing recognition of these isolates as causes of life-threatening invasive fungal infections in patients with hematological disorders mandates knowledge of the microbiology, epidemiology, and options for the prevention and treatment of these previous uncommon opportunistic pathogens (20).

In neutropenic patients with pulmonary infiltrates, the antibiotic treatment should include other antibiotics in addition to the broad-spectrum coverage outlined in Table 1. If a Legionella pneumonia is suspected, erythromycin should be added. The addition of trimethoprim-sulfamethoxazole is indicated if an infection with P. carinii (see Chapter 18) is suspected. Most patients who develop pulmonary infiltrates should also receive empirical antifungal treatment. Patients with hematological neoplasms are also at risk of infection with Mycobacterium tuberculosis and atypical mycobacteriae.

Certain viral pathogens are also responsible for infections in patients with leukemia, lymphoma, and/or neutropenia. Details about herpesvirus infections are given in Chapter 18.

\section{PROPHYLAXIS OF INFECTIONS IN NEUTROPENIC PATIENTS}

A high standard of personal hygiene is essential for the neutropenic patient. If prolonged neutropenia (longer than 3-5 d) is expected, the patient should be housed in private rooms and strict hand washing should be observed. Potential sources of pathogens (e.g., fresh flowers) should be removed from the patient's room. Contact with infected patients should be avoided under all circumstances. For patients with more prolonged neutropenia, such as recipients of bone marrow grafts, special measures such as isolation in rooms with positive pressure and high-efficiency particulate air (HEPA) filtration may reduce the risk of acquisition of aspergillus spores and the development of subsequent invasive aspergillosis (66). The use of a total protective environment (TPE) with isolation of patients in a "sterile" room with HEPA filters and laminar air flow, a low-bacteria diet, topical antiseptics and washings, and the administration of oral nonresorbable antibiotics (colistin, neomycin, norfloxacin) and antifungal agents (amphotericin $B$, nystatin) in these high-risk patients led to the reduction in documented infections in some prospective randomized clinical trials. Unfortunately, the lack of a survival advantage has led to the decline in use of TPE in most centers, although many centers continue to use positive pressure and HEPA filtration. 
Specific antimicrobial prophylaxis has been found to be beneficial in some settings. Numerous studies have focused on the use of oral absorbable antibiotics (e.g., trimethoprim-sulfamethoxazole, erythromycin, fluoroquinolones, and others). The most commonly used agents utilized for antibacterial prophylaxis are the extended-spectrum fluoroquinolones. Although they may reduce the frequency of Gram-negative infections, they do not have significant enough activity to prevent breakthrough infections with Gram-positive organisms and may also lead to the development of resistant Gram-negative organisms. Oral antibiotics, therefore, should be reserved for certain high-risk situations. Antifungal prophylaxis with fluconazole was shown to be highly effective in prevention of infections with Candida (mainly albicans species) in bone marrow transplant recipients in several randomized trials in the 1990s. Recent studies are focusing on the utility of newer broader-spectrum agents such as voriconazole and posaconazole in this high-risk group of patients. Unfortunately, like antibacterial therapy, when an effective therapy for one infection is found (e.g., Aspergillus), increased or "breakthrough" infection with resistant organisms may be seen (e.g., Zygomycetes).

Although previously considered a protozoa, $P$. carinii (also designated as $P$. jarvoii), is now classified as a fungus. Trimethoprim-sulfamethoxazole is active as a prophylaxis of infections with P. carinii. (see Chapter 18). Although aerosolized treatment with pentamidine and oral dapsone has been used, breakthrough infections have been seen. Trimethoprim-sulfamethoxazole therefore remains the prophylactic agent of choice. Such a prophylaxis is indicated in highrisk situations (e.g., bone marrow transplants, patients with acute lymphoblastic leukemia under high-dose steroids).

Antiviral prophylaxis with acyclovir was shown to be extremely effective in reduction of the morbidity due to mucositis exacerbated by infection with H. simplex after bone marrow transplant over two decades ago. Although many centers use acyclovir, valcyclovir, or famciclovir for prophylaxis against infection with $V$. zoster virus, the dose of drug needed is higher and the period of time required for adequate protection is much longer (e.g., 6-12 mo after a hematopoietic stem cell transplant) than that needed for prophylaxis against $H$. simplex. Many clinicians will, therefore, educate their patients, family members, and other caregivers regarding the early signs and symptoms of viral infection. Antiviral treatment for Varicella is then withheld until the earliest signs of infection are evident. This approach is only effective if therapy is begun within 48$72 \mathrm{~h}$ of the first sign of infection and may serve to reduce the risk of visceral dissemination, reducing the need for prolonged prophylaxis in high-risk patients. For CMV infections (see Chapter 18), several drugs are active for prophylaxis (e.g., ganciclovir, foscarnet, cidofovir), but because of their toxicity profile, 
routine prophylactic use is not indicated. Prospective monitoring of blood for a positive signal for CMV antigen or by quantitative PCR is now standard of care for patients at high risk (i.e., allogeneic blood or marrow transplant recipients, patients receiving therapy with anti-CD52 antibody, etc.) for development of CMV-related disease (e.g., pneumonitis, hepatitis, cerebritis). Pre-emptive therapy with either ganciclovir or foscarnet is given in the presence of a positive signal for CMV to reduce the risk of progression to CMV disease and withheld in patients with a negative signal in attempt to avoid unwarranted drug toxicity. Recent and ongoing prophylactic studies of newer oral formulations of antiviral agents with activity against CMV (e.g., valganciclovir) are promising. The routine use of hyperimmuneglobulins as a means of antiviral prophylaxis, more common in past years, has fallen in to disfavor because of costs, toxicities, and the improvements in the use of pre-emptive antiviral therapy.

Many treatment protocols for hematological malignancies include the use of hematopoietic growth factors. These factors (granulocyte colony-stimulating factor [G-CSF], granulocyte/macrophage colony-stimulating factor [GM-CSF], and others) generally shorten neutropenia by several days. The rationale, dosage, and side effects of hematopoietic growth factors are discussed in Chapter 2.

\section{VENOUS ACCESS}

Patients who undergo intensive treatment for hematological malignancies need multiple transfusions, often parenteral nutrition, antibiotics, cytostatic drugs, and multiple other drugs. In these patients, a large lumen venous catheter is indicated. For patients who undergo multiple cycles of chemotherapy (e.g., patients with lymphomas) or who will have prolonged cytopenias (e.g., in bone marrow or stem cell transplantation) or who need prolonged parenteral nutrition, an implanted catheter system is useful (portacath systems, Hickman or Broviac multiple lumen systems, and other catheters). These systems are implanted surgically and can stay for several months if necessary. Portacath-type systems need special needles for access and are recommended for patients who experience shorter cytopenias or who need long-term parenteral nutrition. Hickman-type catheters are suited for patients with acute leukemia or who undergo marrow transplantation. The implanted catheter systems are not free from complications (total rate 2-5\%) including bleeding or pneumothorax at the time of implantation and thromboses or infections later on.

If a central venous catheter is the source of bacteremia, an attempt can be made to treat the infection by appropriate antibiotics including vancomycin. If the fever or the local signs of infection do not regress within $24 \mathrm{~h}$, the catheter should be replaced. The catheter should be drawn immediately if signs of a tunnel infection are evident. 
Table 3

Antiemetic Regimen for Chemotherapy in Leukemias and Lymphomas

\begin{tabular}{ll}
\hline Regimen & Dose \\
\hline A. Ondansetron + & $2 \times 8 \mathrm{mg} \mathrm{i.v.}$ \\
$\quad$ Dexamethasone & $10 \mathrm{mg} \mathrm{i.v.}$ \\
B. Ondansetron + & $2 \times 8 \mathrm{mg} \mathrm{i.v.}$ \\
$\quad$ Dexamethasone + & $5 \mathrm{mg} \mathrm{i.v.}$ \\
$\quad$ Aprepitant & $125 \mathrm{mg} \mathrm{p.o.} \mathrm{d} 1,80 \mathrm{mg} \mathrm{d} \mathrm{2,3} 3$ \\
C. Ondansetron & $2 \times 8 \mathrm{mg} \mathrm{i.v.}{ }^{a} b$ \\
D. Metoclopramide \pm & $3 \times 1-2 \mathrm{mg} / \mathrm{kg}^{b}$ \\
$\quad$ Dexamethasone & $10 \mathrm{mg} \mathrm{i.v.}$ \\
\hline
\end{tabular}

\footnotetext{
${ }^{a}$ First dose $1 \mathrm{~h}$ before chemotherapy, second and third dose after 4 and $8 \mathrm{~h}$, intravenously (i.v.) or orally.

${ }^{b}$ First dose $1 \mathrm{~h}$ before chemotherapy, second and third dose after 2 and 4 h i.v.
}

A local thrombus in a central venous catheter can often be reopened by injecting $1-3 \mathrm{~mL}$ of a solution containing $3000-5000 \mathrm{U} / \mathrm{mL}$ of streptokinase or urokinase. The extravasation of cytostatic drugs through a central venous catheter that has been correctly positioned is almost impossible. However, if this occurs as a result of leakage of the catheter, the affected tissue should be infiltrated with 5$15 \mathrm{~mL}$ of isotonic saline solution and the catheter should he removed. Necrotic tissue should be excised surgically.

\section{FURTHER CONSIDERATIONS}

All patients with hematological malignancies need an intensive emotional support. Many patients suffer from anxiety, whereas others develop major depressions or other affective disorders requiring treatment.

Many patients need analgesia. The side effects of commonly used analgesic drugs (e.g., respiratory depression with morphine) and drug interactions must be considered.

Nausea and vomiting are common side effects of many cytostatic drugs and can be prevented or ameliorated by modern antiemetic therapy. Examples of effective regimens are given in Table 3; however, they must be modified individually. The choice of antiemetic agent depends on the emetogenic potential of the chemotherapy. Several other emetic drugs have a similar mechanism of action. The side effects of these antiemetic drugs should be considered. Ondansetron belongs to the class of 5-hydroxytryptamine antagonists and has only minor side effects (headaches, slight elevation of transaminases). Palonosetron (recommended dose $0.25 \mathrm{mg}$ i.v.) is a longer acting 5-hydroxytryptamine antago- 
nist and should be considered in cases of delayed nausea and vomiting. Combinations using aprepitant (a neurokinin-1-receptor antagonist) should be considered in high-emetogenic chemotherapy; however, the dose of dexamethasone (if part of the regimen) should be reduced by half (because dexamethasone is eliminated by CYP3A4).

Metoclopramide is an effective antiemetic agent but has troublesome side effects in 5-20\% of patients (extrapyramidal reactions, dystonic reactions, sedation, diarrhea). Steroids are effective in combination regimens of antiemetic drugs and have few side effects if used on a short-term basis. Other drugs (benzodiazepines, cannabinoids) are also useful and effective in antiemetic combination treatments.

\section{REFERENCES}

1. Bodey GP, Buckley M, Sathe YS, et al. Quantitative relationships between circulating leukocytes and infection in patients with acute leukemia. Ann Intern Med 1966; 64:328-340.

2. Pizzo PA. Fever in immunocompromised patients. N Engl J Med 1999;341:893-900.

3. Donowitz GR, Maki DG, Crnich CJ, Pappas PG, Roloston KV. Infections in the neutropenic patient-new views of an old problem. Hematology 2001;113-139.

4. Hughes WT, Armstrong D, Bodey GP, et al. 2002 guidelines for the use of antimicrobial agents in neutropenic patients with cancer. Clin Infect Dis 2002;34:730-751.

5. de Pauw BE, Donnelly JP. Infections in the immunocompromised host: general principles. In: Mandell GL, Bennett JE, Dolin R, eds. Mandell, Douglas, and Bennett's Principles and Practices of Infectious Diseases, $5^{\text {th }}$ ed. Philadelphia: Churchill Livingston, 2000:pp. 30793090.

6. Bodey GP. Unusual presentations of infection in neutropenic patients. Int J Antimicrob Agents 2000;16:93-95.

7. DesJardin JA, Falagas ME, Ruthazer R, et al. Clinical utility of blood cultures drawn from indwelling central venous catheters in hospitalized patients with cancer. Ann Intern Med 1999;131:641-647.

8. Caillot D, Couaillier J-F, Bernard A, et al. Increasing volume and changing characteristics of invasive pulmonary aspergillosis on sequential thoracic computed tomography scans in patients with neutropenia. J Clin Oncol 2001;19:253-259.

9. Pazos C, Ponton J, Del Palacio A. Contribution of (1-3) beta-D-glucan chromogenic assay to diagnosis and monitoring of invasive aspergillosis in neutropenic adult patients. $J$ Clin Microbiol 2005;43:299-305.

10. Pinel C, Fricker-Hidalgo H, Lebeau B, et al. Detection of circulating Aspergillus fumigatus galactomannan value and limits of the Platelia test for diagnosing invasive aspergillosis. J Clin Microbiol 2003;41:2184-2186.

11. Walsh TJ, Shoham S, Petraitiene R, et al. Detection of galactomannan antigenemia in patients receiving piperacillin-tazobactam and correlations between in vitro, in vivo, and clinical properties of the drug-antigen interaction. J Clin Microbiol 2004;42:4744-4748.

12. Rolston KVI. Stenotrophomonas maltophilia infection in cancer patients-frequency, spectrum of infection and antimicrobial susceptibility. European Congress of Clinical Microbiology and Infectious Diseases (ECCMID) 2003;Glasgow, Scotland Abs. 678 
13. Safdar N, Maki DO. The commonality of risk factors for nosocomial colonization and infection with antimicrobial-resistant Staphylococcus aerues, enterococcus, gram-negative bacilli, Clostridium difficile, and Candida. Ann Intern Med 2002;136:834-844.

14. Razonable RR, Litzow MR, Kaliq Y, et al. Bacteremia due to viridans group streptococci with diminished susceptibility to levofloxacin among neutropenic patients receiving levofloxacin prophylaxis. Clin Infect Dis 2002; 34:1469-1474.

15. Jantunen E, Ruutu P, Niskanen L, et al. Incidence and risk factors for invasive fungal infections in allogeneic BMT recipients. Bone Marrow Transplant 1997;19:801-808

16. Yuen KY, Woo PCY, Ip MSM, et al. Stage-specific manifestations of mold infections in bone marrow transplant recipients: risk factors and clinical significance of positive concentrated smears. Clin Infect Dis 1997;25:37-42.

17. Fukuda T, Boeckh M, Carter RA, et al. Risks and outcomes of invasive fungal infections in recipients of allogeneic hematopoietic stem cell transplants after nonmyeloablative conditioning. Blood 2003;102:827-833.

18. Keating MJ, Flinn I, Jain V, et al. Therapeutic role of alemtuzumab (Campath - $1 \mathrm{H}$ ) in patients who have failed fludarabine: results of a large international study. Blood 2002;99:3554-3561.

19. McNeil MM, Nash SL, Hajjeh RA, et al. Trends in mortality due to invasive mycotic diseases in the United States. 1980-1997. Clin Infect Dis 2001;33:641-647.

20. Walsh TJ, Groll A, Hiemenz J, et al. Infections due to emerging and uncommon fungal pathogens. Clin Microbiol Infect 2004;10:48-66.

21. Ison MG, Hayden FG, Kaiser L, et al. Rhinovirus infections in hematopoietic stem cell transplant recipients with pneumonia. Clin Infect Dis 2003;36:1139-1143.

22. Martino R, Ramila E, Rabella N, et al. Respiratory virus infections in adults with hematologic malignancies: a prospective study. Clin Infect Dis 2003;26:1-8.

23. Marcolini JA, Malik S, Suki D, et al. Respiratory disease due to parainfluenza virus in adult leukemia patients. Eur J Clin Microbiol Infect Dis 2003;22:79-84.

24. Freifeld A, Marchigiani D, Walsh T, et al. A double-blind comparison of empirical oral and intravenous antibiotic therapy for low risk febrile patients with neutropenia during cancer chemotherapy. N Eng J Med 1999;341:305-311.

25. Rolston KVI. New trends in patient management: risk-based therapy for febrile patients with neutropenia. Clin Infect Dis 1999;29:515-521.

26. Del Favero A, Menichetti F, Marino P, et al. A multicenter, double-blind, placebo-controlled trial comparing piperacillin-tazobactam with and without amikacin as empiric therapy for febrile neutropenia. Clin Infect Dis 2001;33:1295-1301.

27. Walsh TJ, Hiemenz JW, Anaissie E. Recent progress and current problems in treatment of invasive fungal infections in neutropenic patients. Infect Dis Clin North Am 1996;10:365-400.

28. Pizzo PA, Robichaud KJ, Gill FA, et al. Empiric antibiotic and antifungal therapy for cancer patients with prolonged fever and granulocytopenia. Am J Med 1982;72:101-111.

29. EORTC International Antimicrobial Therapy Cooperative Group. Empiric antifungal therapy in febrile granulocytopenic patients. Am J Med 1989;86:668-672.

30. White MH, Bowden RA, Sandler ES, et al. Randomized, double-blind clinical trial of amphotericin B colloidal dispersion vs. amphotericin B in the empirical treatment of fever and neutropenia. Clin Infect Dis 1998;27:296-302.

31. Walsh TJ, Finberg RW, Arndt C, et al. Liposomal amphotericin B for empirical therapy in patients with persistent fever and neutropenia. N Engl J Med 1999; 340:764-771.

32. Boogaerts M, Winston DJ, Bow EJ, et al. Intravenous and oral itraconazole versus intravenous amphotericin B deoxycholate as empirical antifungal therapy for persistent fever in neutro- 
penic patients with cancer who are receiving broad-spectrum antibacterial therapy; a randomized controlled trial. Ann Intern Med 2001;135:412-422.

33. Walsh TJ, Pappas P, Winston DJ, et al. Voriconazole compared with liposomal amphotericin $\mathrm{B}$ for empirical antifungal therapy in patients with neutropenia and persistent fever. $\mathrm{N} \mathrm{Engl}$ J Med 2002;346:225-234.

34. Walsh TJ, Teppler H, Donowitz GR, et al. Caspofungin versus liposomal amphotericin B for empirical antifungal therapy in patients with persistent fever and neutropenia. $N$ Engl J Med 2004;351(14):1391-1402.

35. National Nosocomial Infections Surveillance System. National Nosocomial Infections Surveillance (NNIS) System report. Data summary from January 1990-May 1999. Am J Infect Control 1999;27:520-532.

36. National Nosocomial Infections Surveillance System. National Nosocomial Infections Surveillence (NNIS) System report. Data summary from January 1992-April 2000. Am J Infect Control 2000;28:429-448.

37. Tunkel AR, Sepkowitz KA. Infections caused by viridans streptococci in patients with neutropenia. Clin Infect Dis 2002;34:1524-1529.

38. Donskey CJ, Chowdhry TK, Hecker MT, et al. Effect of antibiotic therapy on the density of vancomycin-resistant enterococci in the stool of colonized patients. $N$ Engl J Med 2000;343:1925-1932.

39. Edmond M, Ober JF, Weinbaum DL, et al. Vancomycin-resistant Enterococcus faecium bacteremia: risk factors for infection. Clin Infect Dis 1995;20:1126-1133.

40. Patterson DL, Rice LB. Empirical antibiotic choice for the seriously ill patient; are minimization of selection of resistant organisms and maximization of individual outcome mutually exclusive? Clin Infect Dis 2003;36:1006-1012.

41. National Nosocomial Infections Surveillance System. National Nosocomial Infections Surveillance (NNIS) System report. Data summary from January 1992-June 2001. Am J Infect Control 2001;29:404-421.

42. Stevens DL, Dotter B, Madaras-Kelly K. A review of linezolid: the first oxazolidinone antibiotic. Expert Rev Anti Infect Ther 2004;2:51-59.

43. Raad I, Hachem R, Hanna H, et al. Prospective randomized study comparing quinupristin with linezolid in the treatment of vancomycin-resistant Enterococcus faecium infections. $J$ Antimicrob Chemother 2004;53:646-649.

44. Rao N, Ziran BH, Wagener MM, et al. Similar hematologic effects of long-term linezolid and vancomycin in a prospective observational study of patients with orthopedic infections. Clin Infect Dis 2004;38:1058-1064.

45. Meissner HC, Townsend T, Wenman W, et al. Hematologic effects of linezolid in young children. Pediatr Infect Dis J 2003;22(S19):186-192.

46. Dibo I, Pillai SK, Gold HS, et al. Linezolid-resistant Enterococcus faecalis isolated from a cord blood transplant recipient. J Clin Microbiol 2004;42:1843-1845.

47. Pappas PG, Rex JH, Sobel JD, et al. Guidelines for the treatment of candidiasis. Clin Infect Dis 2004;38:161-189.

48. Walsh TJ, Hiemenz JW, Anaissie E. Recent progress and current problems in treatment of invasive fungal infections in neutropenic patients. Infect Dis Clin North Am 1996;10:365-400.

49. Nucci M, Anaissie E. Should vascular catheters be removed from all patients with candidemia? An evidence-based review. Clin Infect Dis 2002;34:591-599.

50. Walsh TJ, Rex JH. All catheter-related candidemia is not the same: assessment of the balance between the risks and benefits of removal of vascular catheters. Clin Infect Dis 2002;34:600-602. 
51. Mora-Duarte J, Betts R, Rotstein C, et al. Comparison of caspofungin and amphotericin B for invasive candidiasis. N Engl J Med 2002;347:2020-2029.

52. Gerson SL, Talbot GH, Hurwitz S, et al. Prolonged granulocytopenia: the major risk factor for invasive pulmonary aspergillosis in patients with acute leukemia. Ann Intern Med 1984;100:345-351.

53. Patterson TF, Kirkpatrick WR, White M, et al. Invasive aspergillosis. Disease spectrum, treatment practices, and outcomes. Medicine (Baltimore) 2000;79:250-60.

54. Stevens DA, Kan VL, Judson MA, et al. Practice guidelines for diseases caused by Aspergillus. Clin Infect Dis 2000;30:696-709.

55. Hiemenz JW, Walsh TJ. Lipid formulations of amphotericin B: Recent progress and future directions. Clin Infect Dis 1996;22(S2):133-144.

56. Herbrecht R, Denning DW, Patterson TF, et al. Voriconazole versus amphotericin B for primary therapy of invasive aspergillosis. N Engl J Med 2002;347:408-415.

57. Caillot D, Mannone L, Cuisenier B, et al. Role of early diagnosis and agressive surgery in the management of invasive pulmonary aspergillosis in neutropenic patients. Clin Microbiol Infect 2001;7(S2):54-61.

58. Bartizal K, Gill CJ, Abruzzo GK, et al. In vitro preclinical evaluation studies with the echinocandin antifungal MK-0991 (L-743,872). Antimicrob Agents Chemother 1997;41: 2326-2332.

59. Arikan S, Lozano-Chiu M, Paetznick V, et al. In vitro synergy of caspofungin and amphotericin B against Aspergillus and Fusarium spp. Antimicrob Agents Chemother 2002;46:245-247.

60. Perea S, Gonzalez G, Fothergill AW, et al. In vitro interaction of caspofungin acetate with voriconazole against clinical isolates of Aspergillus spp. Antimicrob Agents Chemother 2002;46:3039-3041.

61. Kirkpatrick WR, Perea S, Coco BJ, et al. Efficacy of caspofungin alone and in combination with voriconazole in a guinea pig model of invasive aspergillosis. Antimicrob Agents Chemother 2002;46:2564-2568.

62. Petraitis V, Petraitiene R, Sarafandi AA, et al. Combination therapy in treatment of experimental pulmonary aspergillosis: synergistic interaction between an antifungal triazole and an echinocandin. J Infect Dis 2003;187:1834-1843.

63. Aliff TB, Maslak PG, Jurcic JG, et al. Refractory aspergillus pneumonia in patients with acute leukemia. Successful therapy with combination caspofungin and liposomal amphotericin. Cancer 2003;97:1025-1032.

64. Kontoyiannis DP, Hachem R, Lewis RE, et al. Efficacy and toxicity of caspofungin in combination with liposomal amphotericin $\mathrm{B}$ as primary or salvage treatment of invasive aspergillosis in patients with hematological malignancies. Cancer 2003;98:292-299.

65. Marr KA, Boeckh M, Carter RA, et al. Combination antifungal therapy for invasive aspergillosis. Clin Infect Dis 2004;39:797-802.

66. Sherertz RJ, Belani A, Kramer BS, et al. Impact of air filtration on nosocomial aspergillus infections. Unique risk of bone marrow transplant recipients. Am J Med 1987;83:7109-7718. 